\title{
Supernumerarios múltiples no sindrómicos en dentición permanente. Reporte de caso
}

\author{
Multiple non syndromic supernumerary teeth in permanent dentition. A case report \\ Pedro Martin Jesús Aparcana-Quijandría, ${ }^{1, a}$, José Luis Huamani-Echaccaya ${ }^{1,2, b}$
}

\section{RESUMEN}

El número de dientes puede incrementarse por piezas dentarias denominadas Dientes Supernumerarios (DS). En los casos en que los DS son cinco o más y no relacionados con ningún síndrome, son considerados DS múltiples o hiperdoncia múltiple no sindrómica; anomalía dentaria con una escasa prevalencia menor al 1\% de los reportes de esta alteración. El objetivo de esta investigación es documentar un caso poco frecuente de DS múltiples no sindrómicos en una paciente con dentición permanente. Paciente de sexo femenino, 22 años de edad, presenta siete DS en total; clínicamente: dos DS en la zona central superior, un DS por palatino de la pieza 1.2, un DS por distal de la pieza 3.5. Radiográficamente: un DS entre las piezas 1.5 y 1.4, la pieza 1.1 retenida en posición horizontal, la agenesia de la pieza 2.2 y dos DS suplementarios entre los premolares inferiores derechos. El diagnóstico precoz de los DS es fundamental para evitar las alteraciones futuras originadas por esta anomalía dentaria; así como un examen adecuado que permita descartar la asociación con hiperdoncia relacionada a algún síndrome.

PALABRAS CLAVE: Diente supernumerario, síndrome, anomalía dentaria.

\section{SUMMARY}

The number of teeth can be increased by extra teeth named Supernumerary Teeth (ST). Multiple ST or hyperdontia is consider when there are five or ST with non syndromic association, which is a dental anomaly with a prevalence less than $1 \%$ of the reports of this alteration. The aim of this report was to keep record an unusual case of nonsyndromic supernumerary teeth in a permanent dentition patient. Intraoral examination of a 22 years old woman revealed permanent dentition with presence of two supernumerary teeth in the upper central region, one of them palatally to 1.2 , and another distal to 3.5. Radiographic findings: A panoramic radiograph showed us impacted supernumerary teeth between 1.5 and 1.4, another in similar condition, 1.1 in horizontal position, agenesy of 2.2 tooth, and two supernumeraries more between right lower bicuspids. The early diagnosis of ST is essential to avoid future alterations caused by this dental anomaly; as well as an adequate examination that allows to rule out the association with hyperdoncia related to some syndrome.

KEY WORDS: Tooth, supernumerary, syndrome, tooth abnormalities

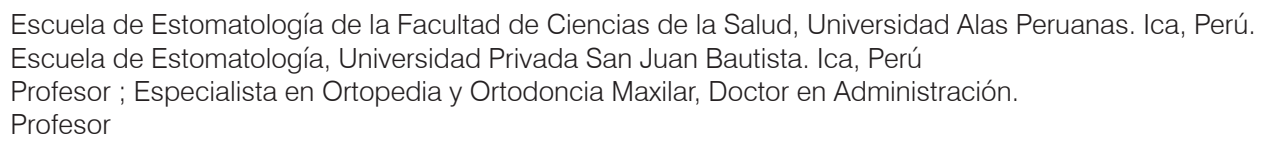




\section{INTRODUCCIÓN}

Durante el proceso de desarrollo de la dentición humana se puede presentar alteraciones de forma, tamaño, erupción, posición y número de piezas dentarias. En cuanto al número estas alteraciones pueden presentarse por disminución en la cantidad de piezas dentarias, denominado hipodoncia o por exceso de piezas dentarias conocido como hiperdoncia. $(1,2,3)$, presentando ambas una prevalencia en conjunto del $12.7 \%$ de la población (4).

El incremento en el número de piezas dentarias se presenta a través de dientes supernumerarios (DS) únicos, alteración denominada hiperdoncia simple o con el desarrollo de varios DS denominada hiperdoncia múltiple no sindrómica(5). Esta alteración presenta poca prevalencia el diagnóstico se sustenta por la presencia de cinco o más DS, indistintamente de su disposición, además de la ausencia de alguna anomalía concomitante que influya en el incremento en el número de dientes (6).

A través de las investigaciones se atribuye una etiología multifactorial a los DS, dentro de estas se mencionan la involución filogenética (atavismo), la división o dicotomía del germen dental, las variaciones de la organogénesis, el incremento de la actividad de la lámina dentaria, factores ambientales, hereditarios y genéticos $(7,8)$.

La prevalencia de DS varia notablemente entre la dentición decidua y la dentición permanente ; así como entre las poblaciones estudiadas; se calcula un intervalo entre $0,3-0,6 \%$ en la dentición decidua y de 0,5 a $3,8 \%$, para la dentición permanente $(4,9)$. La hiperdoncia simple es la más frecuente presentándose entre el $76 \%$ a $86 \%$ de la casuística; en cuanto a la hiperdoncia múltiple, la presencia de dos DS se reporta entre el $12 \%$ a $25 \%$ de los pacientes $(10,11)$ la casuística mayor a cinco o más DS en pacientes no sindrómicos es muy poco frecuente con una prevalencia menor al $1 \%$ de los casos $(9,12)$ situación sostenida con los reportes y la alta prevalencia de casos de DS asociados con síndromes como labio y paladar hendido, Displasia Cleidocraneal y el Síndrome de Gardner $(11,13,14)$. De acuerdo a múltiples estudios, la prevalencia de DS es mayor para el sexo masculino que el femenino, en una proporción de 2:1 respectivamente $(11,15,16)$.
Los DS también han sido agrupados y clasificados en cuanto a su forma; siendo aquellos que guardan similitud con la pieza dentaria de la secuencia normal denominados como eumórficos o suplementarios. Así mismo los DS pueden presentar una morfología diferente a los dientes de la secuencia normal, siendo denominados como dismórficos; este tipo de DS presenta diversas morfologías dentro de los cuales se encuentran los conoides de forma de cono y raíz corta, los tuberculados que suelen ser multicuspídeos y de raíz con proporciones casi siempre adecuadas y los rudimentarios que no presentan forma definida (17). En cuanto a la frecuencia de la forma, los cónicos son los más comunes con $39 \%$, seguido de los rudimentarios con $27.5 \%$, los suplementarios con $25.6 \%$ y los tuberculados con $8 \%(11,18)$. La localización de los DS también es variada; la más frecuente es en la región premaxilar 52,82\% seguido de la región premolar inferior $29,44 \%$, la región molar superior $7,67 \%$, la región premolar superior $4,03 \%$, la región molar inferior $4,03 \%$, y, finalmente, la región antero inferior $2,02 \%(4,19)$.

Las alteraciones que ocasionan los DS se manifiestan en diversos trastornos como retención prolongada de los dientes temporales, modificación en la erupción de los dientes permanentes, ectopia, apiñamiento, diastemas, formación de quistes, reabsorción radicular de las piezas contiguas y estética (20). Por lo general el tratamiento de los DS es la exodoncia, sin embargo, sus características, posición, así como su relación con la oclusión y piezas dentarias proximales amerita un tratamiento

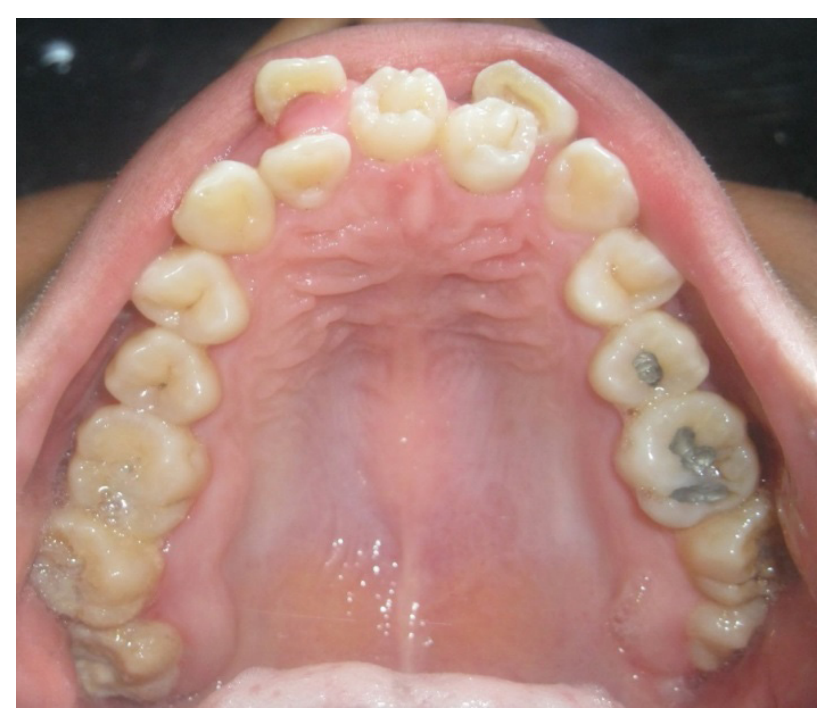

Figura 1. Vista oclusal del maxilar superior 
integral el cual no debe de ser subestimado $(11,17)$. El objetivo de esta investigación es reportar un caso clínico poco frecuente de múltiples DS -siete en total - que se producen sin ninguna asociación sindrómica en una paciente de sexo femenino con dentición permanente.

\section{Reporte de caso}

Paciente de sexo femenino, de 22 años de edad, procedente de la ciudad de Ica, acudió a consulta por "sentir vergüenza al hablar y sonreír" previo a las evaluaciones se diligenció el consentimiento informado, procediendo al examen ectoscópico, presentó fascie asimétrica, perfil anteroposterior convexo. Al examen regional estomatológico, presentó labios hidratados gruesos, ausencia de cierre labial, saliva abundante y viscosa.

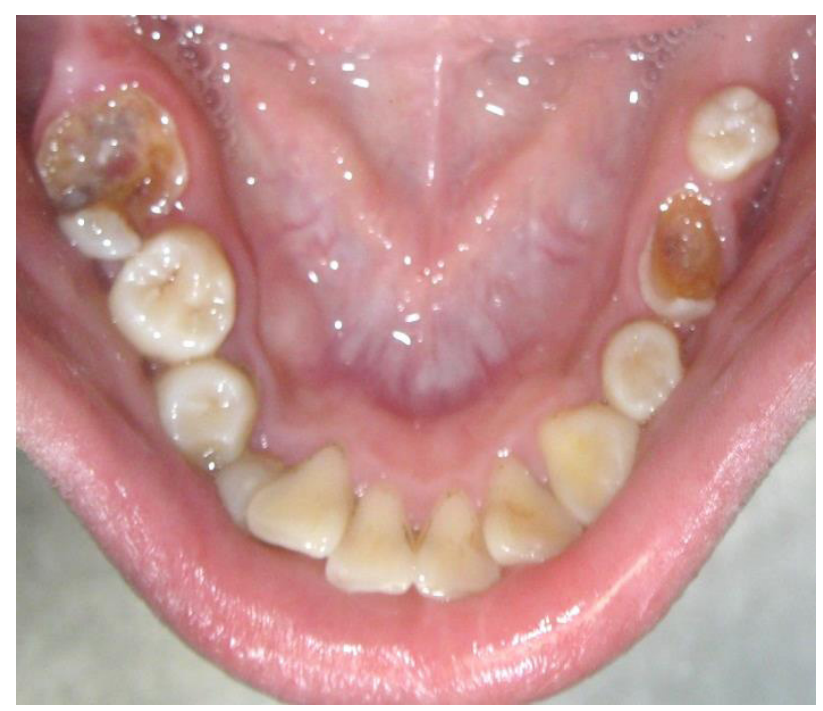

Figura 2. Vista oclusal del maxilar inferior

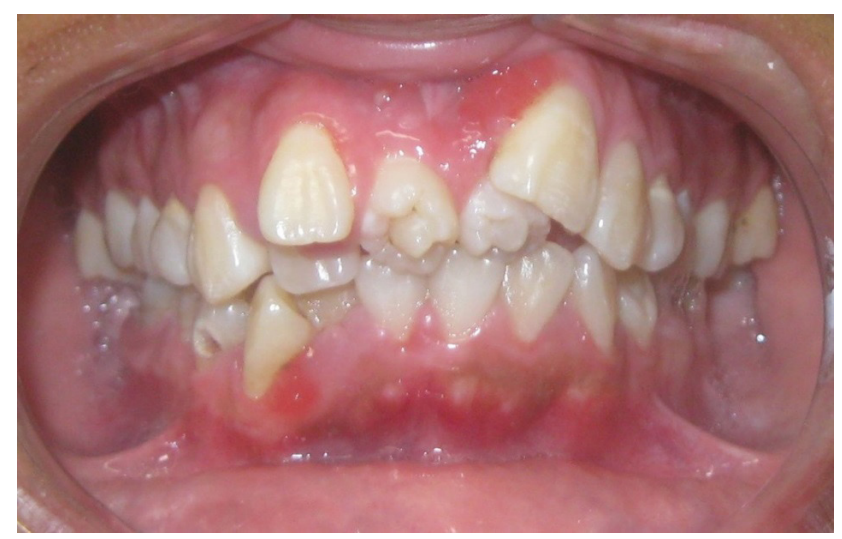

Figura 3. Vista frontal arcadas en oclusión
Al examen clínico intraoral el maxilar superior presenta dos DS en la zona central de caras redondeadas y multicuspídeas ubicadas entre la pieza 1.2 y 2.1; ausencia clínica de la pieza 1.1; un tercer DS de tipo suplementario orientado verticalmente y ubicado por palatino de la pieza 1.2; ausencia de la pieza 2.2, apiñamiento generalizado, giroversión de los primeros y segundos premolares y múltiples lesiones cariosas en ambos cuadrantes. (figura 1). En cuanto al maxilar inferior presenta un cuarto DS de tipo tuberculado ubicado por distal de la pieza 3.5 la cual se encuentra con giroversión en sentido mesial y una amplia destrucción coronaria; apiñamiento anterior, giroversión de premolares en el tercer y cuarto cuadrante, ausencia de las piezas 3.7, 3.6, 4,7 y destrucción coronaria de la pieza 4.6 con evidente compromiso pulpar (figura 2). En cuanto a las arcadas en oclusión en la vista anterior se aprecia el contacto oclusal entre los tres DS anteriores y las piezas antero inferiores, la inclinación hacia mesial del canino derecho, el apiñamiento superior e inferior, la alteración del over bite, over jet y línea media,

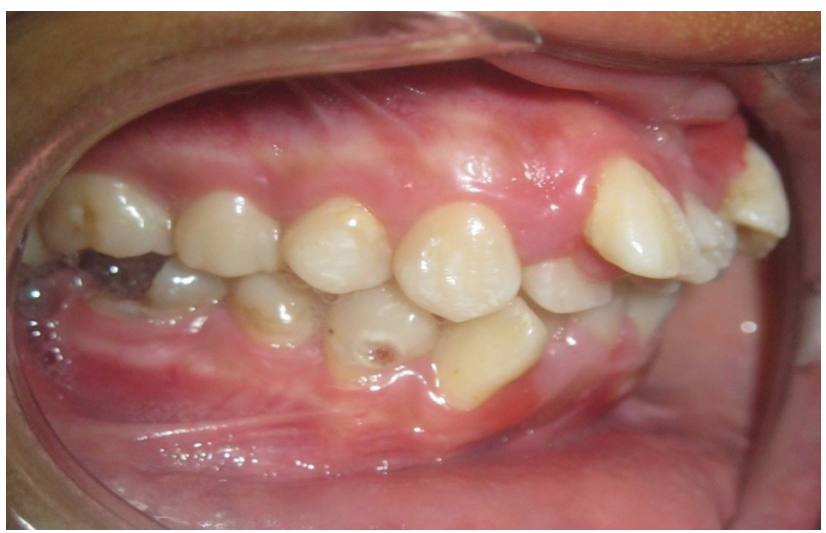

Figura 4. Vista lateral derecha arcadas en oclusión

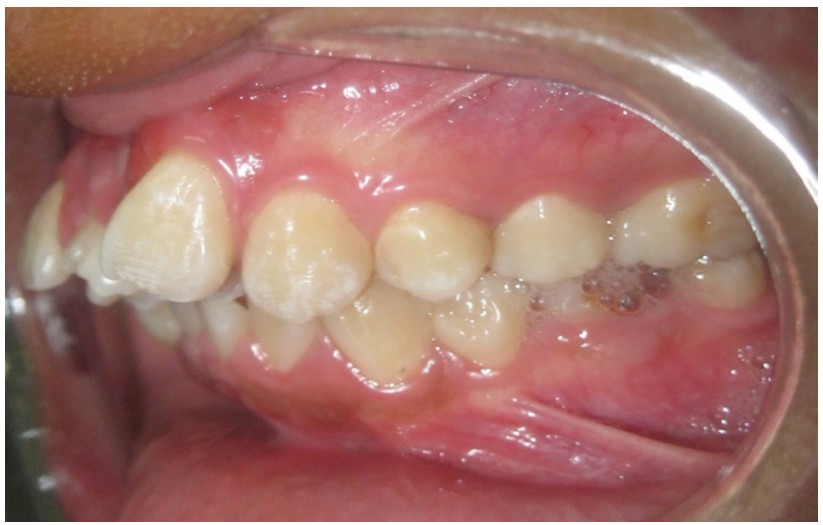

Figura 5. Vista lateral izquierda arcadas en oclusión 
así como la gingivitis localizada. (figura 3). En las vistas laterales derecha e izquierda en oclusión se observa las relaciones molares derecha e izquierda no registrables; la relación canina derecha clase I, mientras que la relación canina izquierda es de clase II (figura 4 y figura 5).

La radiografía panorámica muestra en el maxilar superior un quinto DS con forma de premolar, retenido entre las piezas 1.5 y 1.4 , presenta la pieza 1.1 retenida en posición horizontal con la corona orientada hacia la línea media y la raíz a nivel de los ápices de las piezas $1.3,1.2$, se evidencian los dos DS anterosuperiores de raíces robustas y el DS suplementario por palatino de la pieza 1.2; así como la agenesia de la pieza 2.2. En el maxilar inferior presenta un sexto DS con forma de premolar retenido entre las piezas 4.3 y 4.4, adicionalmente un séptimo DS el cual se encuentra retenido entre las piezas 4.4 y 4.5 y también presenta forma de premolar. Así mismo el DS por distal de la 3.5 ya descrito, el compromiso pulpar de la $3.5 \mathrm{y}$ 4.6, se aprecia la pieza 4.8 semiretenida en posición vertical, la ausencia por exodoncias de las piezas 3.7, 3.6, 4.7; la cabeza de los cóndilos derecho e izquierdo conformación redondeada, ambos senos maxilares neumatizados y los ápices de las piezas dentarias próximos a estos (figura 6).

En cuanto al diagnóstico diferencial que permita determinar una hiperdoncia no sindrómica, se realizó una interconsulta médica, la paciente fue sometida a un examen completo, en el cual se descartó la presencia del síndrome de Gardner, así como de la displasia cleidocraneal, siendo ambos las anomalías más comunes de hiperdoncia múltiple.

\section{DISCUSIÓN}

La presencia de DS que carecen de asociación a algún trastorno sindrómico es poco frecuente, la cantidad de reportes no son numerosos y presentan una menor casuística conforme la cantidad de DS aumenta, siendo la hiperdoncia múltiple con un número mayor a cinco piezas dentarias reportada solo en menos del $1 \%$ de los casos $(9,12)$ situación que describe la singularidad de este estudio ya que cuenta con siete DS de diferente morfología, estado de erupción y dispersa ubicación en ambos maxilares; además de la agenesia de la pieza 2.2.

Los DS únicos son alteraciones un tanto comunes del número de la formula dentaria, sobre todo en la dentición permanente $(1,2)$ sin embargo, no es frecuente el desarrollo de DS múltiples menos aun en pacientes que no presentan síndromes $(11,13,14)$.

Esta alteración, en el número de dientes, presenta una prevalencia con una proporción de 2:1 para el sexo masculino $(11,15,16)$ situación que ratifica lo inusual de este caso al ser la paciente de sexo femenino y presentar DS múltiples. (4).

A pesar de la prevalencia en la casuística reportada, este caso no cuenta con ninguno DS cónico, los cuales son los más comunes con el $39 \%$ de los casos $(5,6)$ por el contrario presenta dos DS tuberculados en la

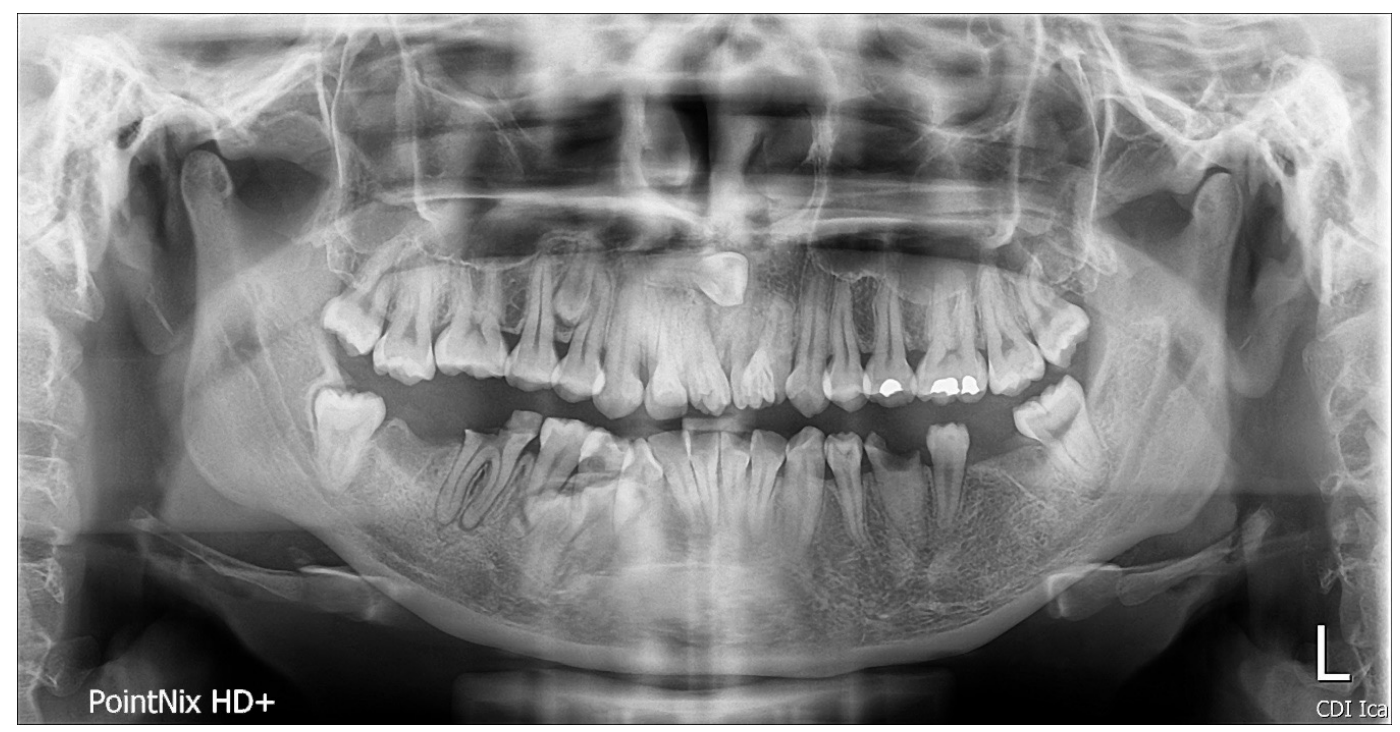

Figura 6. Radiografía panorámica 
zona incisiva anterosuperior; que por lo general solo se aprecian únicos, en la zona premolar en el $8 \%$ y en el $75 \%$ de los casos no erupcionan $(4,19)$ además de presentar un DS suplementario por palatino de la 1.2, otro DS suplementario ubicado entre la 4.4 y 4.5 , además de tres suplementarios retenidos en las zonas premolares superiores e inferiores del lado derecho.

Los DS en cuanto a su forma en este caso, se presentaron como eumórficos erupcionados y no erupcionados, así como dismórficos erupcionados, situación que plantea el caso como muy particular y contrario a la mayoría de los reportes $(11,18)$.

También es de resaltar que este caso contiene varias de las complicaciones de los DS descritas, dentro de estas, los trastornos en la erupción de los dientes permanentes que se refleja en la retención en sentido horizontal de la 1.1, la ectopia de piezas dentales generada en la distalización de la pieza 3.5, el apiñamiento en ambos maxilares, y el deterioro de la estética dental y facial $(11,20)$.

\section{CONCLUSIONES}

El diagnostico de los DS requiere el desarrollo de una historia clínica adecuada, una evaluación exhaustiva tanto clínica como radiológica, así como la realización de la misma lo más temprano posible, ya que repercute directamente en las alteraciones y complicaciones que posteriormente pueden generar requerimientos ortodóncicos, patologías dentales y afectaciones estéticas.

En el caso presentado se realizaran las exodoncia de los DS tuberculados y suplementario ubicados en la zona anterosuperior ya que alteran la estética facial, la oclusión y la autoestima de la paciente; así mismo se realizara la exodoncia de los DS suplementarios que se encuentran retenidos en la zona premolar superior e inferior del lado derecho con la finalidad de eliminar las interferencias para posteriormente previa evaluación de la Especialidad de Ortodoncia se decidirá el tratamiento indicado.

\section{Correspondencia}

Pedro Aparcana Quijandría

Urbanización Santa María H-133. Ica, Ica, Perú.

Correo electrónico: pedroaparcana@hotmail.com

\section{REFERENCIAS BIBLIOGRÁFICAS}

1. Guttal KS, Naikmasur VG, Bhargava P, Bathi RJ. Frequency of developmental dental anomalies in the Indian population. Eur J Dent. 2010; 4(3):263-9. (Citado 15 de julio del 2020) Disponible en: https:// www.ncbi.nlm.nih.gov/pubmed/20613914

2. Neville BW, Damm DD, Allen CM, Bouquot JE. Abnormalities of teeth. In: Oral \& Maxillofacial Pathology. 3rd ed. Philadelphia: W.B. Saunders Company; 2008. (Citado 15 de julio del 2020) Disponible en: https://www.elsevier.com/books/oraland-maxillofacial-pathology/neville/978-1-41603435-3

3. Anthonappa RP, Omer RS, King NM.Characteristics of 283 supernumerary teeth in southern Chinese children. Oral Surg Oral Med Oral Pathol Oral Radiol Endod. 2008;105(6): 48-54. (Citado 15 de julio del 2020) Disponible en: https://www.ncbi.nlm.nih.gov/ pubmed/18417392

4. Baca M. Córdova E. Castillo M. Frecuencia de Anomalías Dentarias de número en radiografías panorámicas de pacientes que asistieron a la Clínica Dental Docente UPCH Sede San Isidro entre los años 2014 y 2017. [Tesis bachiller. Lima, Perú: UPCH; 2018.

5. Batra P, Duggal R, Parkash H. Non-syndromic multiple supernumerary teeth transmitted as an autosomal dominant trait. J Oral Pathol Med. 2005; 34(10):621-5. (Citado 15 de julio del 2020) Disponible en: https:// www.ncbi.nlm.nih.gov/pubmed/16202084

6. Alvira-González J, Gay-Escoda C. Non-syndromic multiple supernumerary teeth: meta-analysis. J Oral Pathol Med. 2012; 41(5):361-6. (Citado 15 de julio del 2020) Disponible en: https://www.ncbi.nlm.nih. gov/pubmed/22092693

7. Abanto J, Imparato JCP, Guedes-Pinto AC, Bönecker M. Anomalías dentarias de impacto estético en odontopediatría: características y tratamiento. Rev Estomatol Herediana. 2012; 22(3) 171-8.

8. Liu D, Zhang W, Zhang ZZ, Wu Y, Ma X. Threedimensional evaluations of supernumerary teeth using cone-beam computed tomography for 487 cases. Oral Surg Oral Med Oral Pathol Oral RadiolEndod. 2007; 103(3): 403-11. (Citado 15 de julio del 2020) Disponible en: https://www.ncbi.nlm.nih.gov/pubmed/17321454

9. Fernández P, Valmaseda E, Berini L, Gay C. Retrospective study of 145 supernumerary teeth. Med Oral Patol Oral Cir Bucal 2006;11:E339-44. (Citado 15 de julio del 2020) Disponible en: https://www.ncbi. nlm.nih.gov/pubmed/16816819

10. Ferrés E, Prats J, Ferrés E. A descriptive study of 113 unerupted supernumerary teeth in 79 pediatric patients in Barcelona.Med. Oral Patol. Oral Cir. Bucal, 2009;14(3):146-52. (Citado 15 de julio del 2020) Disponible en: https://www.ncbi.nlm.nih.gov/ 
pubmed/19242396

11. Aliaga R, Perea M, Aliaga A. Frecuencia de intervenciones quirúrgicas de dientes supernumerarios, odontomas y dientes incluidos en odontopediatría. Rev Estomatol Herediana. 2010; 20(3):196-202. Doi: 10.20453/reh.v20i4.1736

12. Rajab LD, Hamdan MA. Supernumerary teeth: review of the literature and a survey of 152 cases. Int J PaediatrDent. 2002; 12(4):244-54. (Citado 15 de julio del 2020) Disponible en: https://www.ncbi.nlm.nih. gov/pubmed/12121534

13. De Oliveira C, Drummond SN, Jham BC, Abdo EN, Mesquita RA. A survey of 460 supernumerary teeth in Brazilian children and adolescents. Int J PaediatrDent 2008;18:98-106.(Citado 15 de julio del2020)Disponible en: https://www.ncbi.nlm.nih.gov/pubmed/18237292

14. Rao PV, Chidzonga MM. Supernumerary teeth: Literature review. Cent Afr J Med 2001;47:22-6. (Citado 15 de julio del 2020) Disponible en: https:// www.ncbi.nlm.nih.gov/pubmed/11961855

15. Gay C, Mateos M, España A, Gargallo J. Otras inclusiones dentarias. Mesiodens y otros dientes supernumerarios. Dientes temporales supernumerarios. Dientes temporales incluidos. In: Gay C, Aytés L, editors. Tratado de Cirugía Bucal. Madrid: Ergon; 2004. p. 497-534.

16. McNamara CM, Foley TF, Wright GZ, Sandy JR.
The management of premolar supernumeraries in three orthodontic cases. J ClinPediatrDent 1997;22:158. (Citado 15 de julio del 2020) Disponible en: https:// www.ncbi.nlm.nih.gov/pubmed/9643197

17. Garvey MT, Barry HJ, Blake M. Supernumerary teethan overview of classification, diagnosis and management. J Can Dent Assoc. 1999; 65(11): 612-6. (Citado 15 de julio del 2020) Disponible en: https:// www.ncbi.nlm.nih.gov/pubmed/10658390

18. Pérez IE, Chávez AK, Ponce D. Prevalence of supernumerary teeth on panoramic radiographs in a non-adult Peruvian sample. Int. J. Odontostomat. 2014; 8(3):377-383.

19. Szu-Ting C, Hong-Po C, Yi-HsinY, et al. Characteristics of supernumerary teeth among nonsyndromic dental patients. Journal of Dental Sciences. 2015; 10( 2): 133138. (Citado 15 de julio del 2020) Disponible en: https://core.ac.uk/download/pdf/82664433.pdf

20. Chevitarese AB, Tavares CM, Primo L. Clinical complications associated with supernumerary teeth: Report of two cases. J Clin Pediatr Dent 2003;28:27-31. (Citado 15 de julio del 2020) Disponible en: https:// www.ncbi.nlm.nih.gov/pubmed/14604138

Recibido : 15-04-2020

Aceptado : 15-11-2020 